\title{
LEVELS OF MICRO-MEGAKARYOCYTE AND MORTALITY OF PATIENTS WITH MYELODYSPLASTIC SYNDROME
}

\author{
Vera Lady Marlina Sitorus, Zulfikar Lubis, Vinisia Setiadji, Herman Hariman \\ Department of Clinical Pathology, Faculty of Medicine, University of North Sumatra/Adam Malik Hospital, Medan, Indonesia. E-mail: \\ verald406@gmail.com
}

\begin{abstract}
Myelodysplastic Syndrome (MDS) is dysplasia or incorrect growth of blood cells in the bone marrow, and some tend to become malignant. One of the evidence of myelodysplasia is the finding of micro-megakaryocytes in the marrow. Micro-megakaryocytes is a small megakaryocyte with the size of $<40 \mu \mathrm{m}$ and has hypo-granular cytoplasm, with mono- or bi-nuclei. The introduction of micro-megakaryocyte as evidence of MDS is still new and not too many reports about this, however logically micro-megakaryocyte may produce reduce the number and reduced quality platelet. Therefore, this study was designed to investigate whether micro-megakaryocyte may relate to the reduction of platelet number and further to the mortality of the patients. Thirty patients were recruited, but later four were excluded due to loss of follow-up. The remaining 26 cases were investigated where $17 \mathrm{died}$, and nine survive alive. The mean \pm SEM of micro-megakaryocyte of patients who died and still survive are $61.87 \pm 3.43$ and $44.63 \pm 10.28 \%$, respectively $(p<0.01)$. Platelet levels from who died and still survive are $44.23 \pm 13.36$ and $86.67 \pm 39.76(103 / \mu \mathrm{L}) \mathrm{p}<0.01$. This finding showed that the increased level of micro-megakaryocyte could be used as a good prognostic marker for MDS, especially in relation to mortality due to bleeding.
\end{abstract}

Key words: Micro-megakaryocyte, myelodysplastic syndrome

\section{INTRODUCTION}

Myelodysplastic Syndrome (MDS) is dysplasia or incorrect growth in the bone marrow, and some tend to become malignant. Therefore the old term is called preleukemia. As a result of improper growth, MDS may involve dysmyelopoiesis (dysplasia changes in myeloid series), dyserythropoiesis (dysplasia changes in the erythroid series), and dysmegakaryopoiesis (dysplasia changes in megakaryocyte series). ${ }^{1}$

Patients with MDS have varying reductions in the production of red blood cells, platelets, and mature granulocytes that exhibit qualitative defects. These abnormalities often result in anemia, neutropenia, and thrombocytopenia. ${ }^{2}$ Anemia is the most common peripheral blood abnormality, occurring in approximately $80 \%$ to $85 \%$ of patients. Thrombocytopenia occurs in around $30 \%$ to $45 \%$ of MDS cases, about $40 \%$ of patients found to have neutropenia at diagnosis. The impact of thrombocytopenia is often underappreciated, 53\% of patients with MDS experience bleeding, and $25 \%$ experience serious bleeding events during the course of the disease. The clinical manifestation of MDS is nonspecific and variable, depend on MDS subtype, and it ranges from indolent to life threatening. ${ }^{3}$

The World Health Organization (WHO) 2008 classification defines dysmegakaryopoiesis as micro-megakaryopoiesis, hypolobed, or non-lobed nuclei in megakaryocytes of all sizes and multiple, widely-separated nuclei. Although the definition of dysmegakaryopoiesis is potentially useful, there is no precise definition of micro-megakaryocytes in the WHO classification. Micro-megakaryocyte was defined as small megakaryocyte with the size of $<40 \mu \mathrm{m}$ and had hypo-granular cytoplasm, with mono- or bi-nuclei. ${ }^{4}$

\section{METHODS}

Thirty patients with MDS were recruited in this study. Sample collection was based on consecutive recruitment from all patients who underwent bone marrow aspiration procedure in the Department of Clinical Pathology, Division of Hematology, Universitas Sumatera Utara/Adam Malik Hospital, Medan from January 2016-June 2017. Four out of 30 patients were later excluded due to loss to follow-up. The remaining 26 patients were investigated and followed-up. This study received ethical approval 
from the Ethics Committee regarding the implementation of health research activities N0:306/TGL/KEPK FK USU-RSUP HAM/2017, Faculty of Medicine University of North Sumatra.

Venous blood sampling was done such as follows; $3 \mathrm{~mL}$ of the venous blood sample was taken from the median vein. The blood was then put into $3 \mathrm{~mL}$ EDTA vacutainer tube, for the investigation of full blood count.

Bone marrow aspiration was then investigated. The patients were put on the bed, lying on the left side of the body. The area over the right posterior iliac crest and its surrounding were disinfected with $1 \%$ iodine solution and $97 \%$ methanol. The injection of $2 \%$ Lidocaine gave local anesthesia. The marrow aspiration was carried out by Klima needle, where the needle was punctured to the skin and first stopped when it touched the posterior iliac crest. The needle protector was then adjusted and moved forward, assessing the depth of the puncture. The needle was then pushed forward until it stopped by itself when the protector pressed the skin. At that time, the tip of the needle reached the marrow compartment. The trocar was pulled out, and $20 \mathrm{~mL}$ sterile syringe was inserted into the hole. The marrow aspiration took 2-3 mL of marrow aspirate. The aspirate was not mixed with EDTA but spread directly onto the sliding glass to make a marrow film. The specimen was fixed with $97 \%$ methanol for 10 minutes and followed by Giemsa staining for 15 minutes. After drying the slide, the morphology of the bone marrow aspirate was investigated. The percentage of the differential count of the marrow aspirate was calculated from 500 nucleated cells. ${ }^{5}$

Micro-megakaryocyte was defined as small megakaryocyte with the size of $<40 \mu \mathrm{m}$ and had hypo-granular cytoplasm, with mono- or bi-nuclei. Levels of the percentage of micro-megakaryocyte were calculated the same way as the differential count for the morphology of bone marrow aspirate. The calculation was performed in two glass slides, and the result is expressed as a percentage of total megakaryocyte.

$$
\begin{aligned}
& \% \text { megakaryocyte }=\text { micro-megakaryocyte } \\
& \frac{+ \text { other megakaryocytes }}{\text { Total megakaryocyte }} \times 100 \%
\end{aligned}
$$

Sign of myelodysplastic cells was defined based on the proposed Classification of Myelodysplastic Syndrome Cells by The American Society of Hematology 2015, such as evidence of dyserythropoiesis: bizarre nuclei normoblast, inter-cytoplasmic bridging of normoblasts, inter-nucleoplasmic bridging of normoblasts, bi-nucleated normoblasts, fragmented nuclei normoblasts. The proof of dysmyelopoiesis: hypo-or a-granular neutrophils (Pelger-Huet sign), copper-colored neutrophil, dumble-shaped neutrophil, bizarre nuclei neutrophil, hyper-segmented neutrophil ( $\geq 6$ segments). Evidence of dysmegakaryopoiesis were megakaryocyte, single and centralized nuclei megakaryocyte, megakaryocyte with scattered-multiple nuclei (nuclei > 7).

The classification of the myelodysplastic syndrome was stratified based on the proposed classification of the American Society of Hematology 2015. They are: ${ }^{6,7}$ Myelodysplastic Syndrome Single Lineage Dysplasia (MDS SLD) when the dysplasia involves only one line (erythrocyte, neutrophil or thrombocyte); Myelodysplastic Syndrome Multi-Lineage Dysplasia (MDS MLD) when 2 or 3 lines are involved; Myelodysplastic Syndrome with Ring Sideroblast Single Lineage Dysplasia (MDS RS SLD); Myelodysplastic Syndrome with Ring Sideroblast multi Lineage Dysplasia (MDS RS MLD); Myelodysplastic Syndrome with Excess Blast 1 (MDS EB 1) if blast more than $5 \%$ but do not reach more than 10\%; Myelodysplastic Syndrome with Excess Blast 2 (MDS EB 2) when the blast is between 11-19\%; MDS Unclassified.

From the same EDTA tube, the blood undergoes full blood count examination using Sysmex XN-1000. The hemoglobin concentration was tested using the principle of chemical analysis with lauryl sulfate reagent, which is specific for the Sysmex machine and non-cyanide agent. The white blood cell and platelet counting use electric impedance.

Statistical analysis was done with the t-independent test for unpaired data and paired t-test for paired data. A correlation study was performed using the Spearman correlation test.

Mortality of patients is considered as the time from sample collection until the time of the demise of the patients. When patients are still alive, the periods are calculated as the time from sample collection until the date of the last visit to the Hematology Clinic.

\section{RESULTS AND DISCUSSION}

A total of 30 MDS patients were recruited in this study. The comparison of MDS patients male:female 1:2. Reports showed that MDS was found slightly more often in males than females with a ratio $1.4: 1{ }^{8}$ This is different from reports from other countries, the possibility of MDS in Indonesian ethnic is different 
from Mediterranean-Caucasian. Myelodysplastic syndrome is most commonly found in the elderly, with an average age of 70 years. Myelodysplastic syndrome is rarely found before the age of 50 , and if it is so before the age of 50 usually they come from post-radiation or chemotherapy exposure. Contrary in Asia, for an unknown reason, it is more common in the younger age group. ${ }^{9}$ This study shows the mean age of MDS patients are male $43.4+21.35$ years old and female $52.85+13.59$ years old. This result is following Oehadian research. conducted in the Hasan Sadikin Hospital, Bandung who found the mean age of her patients were 48 years with no history of radiation or chemotherapy. ${ }^{10}$

Table 1. The gender and age of the MDS patients

\begin{tabular}{lcc}
\hline Variables & Gender, n(\%) & Age (years) \\
\hline Male & $10(33.33)$ & $43.4 \pm 21.35$ \\
Female & $20(66.67)$ & $52.85 \pm 13.59$ \\
\hline
\end{tabular}

During the two years follow up it was found that $56.67 \%$ (17 patients) died, while 30\% (9 patients) still survives, and loss to follow up $13.33 \%$ (4 patients). In this study, it was confirmed that all patients who died were due to bleeding (17 patients), 2 out of 17 died because of a combination of bleeding and sepsis.

Micro-megakaryocyte was found to be higher in patients who demised, and the platelet was significantly lower in that group (Table 3). On the other hand, the hemoglobin was lower in patients who died, but WBC showed a trend to be higher in patients who died but did not reach statistical significance.

Classically, evolution to $A M L$, infection, and bleeding are reported as the main leading of death. Montoro et al. analyzed causes of death of 200 MDS patients. At the time of analysis, 141 patients (70.5\%) had died; 78 (60.4\%) of the low-risk subgroup and 59 (91\%) of the high-risk subgroup. In the low-risk subgroup, causes of death were an evolution to AML (14), disease progression (5), infection (14), and bleeding (2). In the high-risk subgroup the causes of death were evolution to AML (18), disease progression 3, infection 13 , and bleeding 5 . $^{11}$

Hemoglobin in MDS patients is usually found to be low, this is because that there is some degree of dyserythropoiesis. The dyserythropoietic marrow fails to produce good quality of RBC, and therefore the life span of RBC is short, which make the hemoglobin concentration to below. Moreover, the previous terminology of MDS was named as Jargon as refractory anemia. This fact demonstrates that since the beginning of the history of this disease is always hallmarked with persistent refractory low hemoglobin in spite of the patients are given treatment for their anemia. So, not surprisingly the low hemoglobin in this study is a sign of MDS. ${ }^{3,9}$

The researchers found very interesting data that patients who died with MDS in this study had higher levels of micro-megakaryocyte. This type of megakaryocyte was a small and agranular in characteristic with single lobe. This finding demonstrates that this micro-megakaryocyte had less sufficient capacity for producing a significant number of good quality platelet. The difference of

Table 2. Mortality of the MDS patients

\begin{tabular}{cc}
\hline & Number of patients \\
\hline Mortality : & 17 \\
Mortality caused by bleeding & 15 \\
Mortality due to infection and bleeding & 2 \\
Survive & 9 \\
Lost to follow-up & 4 \\
Total recruited patients & 30 \\
\hline
\end{tabular}

Table 3. Mean+SEM of micro-megakaryocyte, $\mathrm{Hb}, \mathrm{WBC}$, and platelet number in a patient with MDS

\begin{tabular}{lccc}
\hline & Death & Survive & p-value \\
& $\mathbf{n = 1 7}$ & $\mathbf{n = 4}$ & $\mathrm{p}<0.01$ \\
Micro-megakaryocyte $(\%)$ & $61.87 \pm 3.43$ & $44.63 \pm 10.28$ & $\mathrm{p}<0.05$ \\
Hb $(\mathrm{g} / \mathrm{dL})$ & $8.2 \pm 0.62$ & $9.20 \pm 1.19$ & $\mathrm{p}>0.05$ \\
WBC $(/ \mu \mathrm{L})$ & $7.712 \pm 2521$ & $5.324 \pm 995$ & $\mathrm{p}<0.01$ \\
Platelets $(/ \mu \mathrm{L})$ & $44.23 \pm 13.36$ & $86.67 \pm 39.76$ & \\
\hline
\end{tabular}


micro-megakaryocyte between the dead and survive patients showed that it might be possible to use the level of micro-megakaryocyte as a prognostic marker to predict mortality. Since we also found that platelet level was also lower in dead patients that come synchronically with an increased level of micro-megakaryocyte. We can postulate that the cause of death of patients with an increased level of micro-megakaryocyte is caused by the low level of platelets, hence bleeding is the cause of death. Many investigators also reported the cause of death in the majority of DMS patients. This was the first report to demonstrate that there was an association between level of micro-megakaryocyte and low level of platelet that produces the death of MDS patients. ${ }^{12}$

No significant difference in the levels of WBC between the dead and survive MDS patients did not mean that infection was not found in MDS. There was a trend of increase of WBC in dead cases but did not reach statistical significance, and even in our study two cases were shown to be septic as well as in combination with thrombocytopenia as the cause of death.

\section{CONCLUSIONS AND SUGGESTION}

Based on the study, it can be concluded that levels of micro-megakaryocyte is an excellent prognostic marker for patients with MDS and might be related to bleeding. The researchers recommend that micro-megakaryocyte should be calculated the same way as calculating differential count to make clinician aware of the possibility of worse prognosis.

\section{REFERENCES}

1. Liang C, Li J, Cheng J, Chen S, Zhang F, et al. Characteristic of bone marrow cell dysplasia and its effectiveness in diagnosing myelodysplastic syndrome. Pubmed. Hematology Journal, 2018; 23: 65-76.

2. Aster JC, Stone RM. Clinical manifestation and diagnosis of myelodysplastic syndrome. 2017. Availableat https: / / www.uptodate.com/contents / clinical-manifestations-and-diagnosis-of-the-myelodysplastic-syndromes

3. Foran JM, Shammo JM. Clinical presentation, diagnosis, and prognosis of myelodysplastic syndromes. The American Journal of Medicine, 2012; 125(7): 6-13.

4. Feng G, Gale RP, Wen Cui, Wenyu Cai, Gang Huang, et al. A systematic classification of megakaryocytic dysplasia and its impact on prognosis for patients with myelodysplastic syndromes. Exp Hematol Oncol, 2016; 5: 12.

5. Torlakovic EE. ICSH guidelines for the standardization of bone marrow immunohistochemistry. Pubmed Int J Lab Hematol, 2015; 37(4): 431-49.

6. Garcia-Manero G. Myelodysplastic syndromes: 2015 Update on diagnosis, risk-stratification, and management. Am J Hematol, 2015; 90(9): 831-41.

7. Arber DA. The 2016 revision to the World Health Organization classification of myeloid neoplasms and acute Leukemia. Blood, 2016; 127(20): 2391-2405.

8. Neukirchen J, Schoonen WM, Strupp C, Gatterman N, Aul $C$, et al. Incidence and prevalence of myelodysplastic syndromes: Data from Dusseldorf MDS-registry. Leukemia Research, 2011; 35(12): 1591-1596.

9. Fenaux P, Haase D, Sanz GF, Santini V, Buske C. Myelodysplastic syndromes: ESMO clinical practice guidelines for diagnosis, treatment, and follow-up. Ann Oncol, 2014; 25 Suppl 3: 57-69

10. Oehadin A, Fianza PI, Fadjari TH, Sumantri R, Supandiman I. Karakteristik penderita sindroma mielodisplasi di sub bagian hematologi-onkologi medik bagian penyakit dalam RS Hasan Sadikin Bandung selama 5 tahun (1997-2001). Available at http://docplayer.info/48179140-Amaylia-oehadianpandji-irani-fianza-trinugroho-heri-fadjari-rachmatsumantri-iman-supandiman.html

11. Montoro J, Vallespi T, Sancho E, Salamero O, Lopez-Andreoni $L$, et al. Study of causes of death in patients with myelodysplastic syndrome: A single institution experience. Blood, 2011; 118: 5026.

12. Feng G, Cui W, Cai W, Qin T, Zhang Y, et al. Impact of megakaryocyte morphology on prognosis of persons with myelodysplastic syndromes. Blood, 2015; 126: 2876. 